\title{
Enjeux de conservation des endémiques chasmophytes de basse altitude en Méditerranée : cas du genre Silene section Siphonomorpha (Caryophyllaceae) en Algérie
}

\section{Conservation issues of the endemic chasmophytic species of low altitude in the Mediterranean: case of the genus Silene section Siphonomorpha (Caryophyllaceae) in Algeria}

\author{
Manuscrit reçu le 30 novembre 2020 et accepté le 17 mars 2021 \\ Melilia MESBAH ${ }^{* 1}$, Farid BEKDOUCHE ${ }^{1,2}$, Keyssa LAIDI $^{3}$, Errol VÉLA ${ }^{4}$ et \\ Mohamed SAHNOUNE ${ }^{1}$
}

\footnotetext{
${ }^{1}$ Laboratoire d'Ecologie et Environnement, Faculté des Sciences de la Nature et de la Vie, Université de Bejaia, 06000 Bejaia, Algérie.

${ }^{2}$ Département d'Ecologie et Environnement, Faculté des Sciences de la Nature et de la Vie, Université de Batna II. 5000 Batna, Algérie.

${ }^{3}$ Département des Sciences Agronomiques, Faculté des Sciences Biologiques et des Sciences Agronomiques, Université Mouloud Mammeri de Tizi Ouzou, 15000 Tizi Ouzou, Algérie.

${ }^{4}$ AMAP, Université de Montpellier / CIRAD / CNRS / INRA / IRD, CIRAD TA/A51 - PS2. 34398 Montpellier cedex 5, France.
}

\section{Résumé}

Les falaises calcaires méditerranéennes de basse altitude sont connues pour leur diversité et leur richesse exceptionnelles en endémiques, souvent insuffisamment évaluées en Algérie. Cette étude est une analyse et une synthèse des évaluations UICN individuelles de trois endémiques chasmophytes algériennes, Silene sessionis, $S$. aristidis et $S$. auriculifolia. L'objectif est de comprendre les diverses menaces qui s'exercent sur ce type d'habitat et les enjeux de conservation que représentent les espèces qui l'habitent. L'évaluation de celles-ci a été réalisée dans cinq zones littorales ou sublittorales du pays. Leurs statuts de conservation ont été établis selon les recommandations de l'UICN et validés par le processus officiel de Liste Rouge. De nouveaux sites ont été découverts pour $S$. sessionis et $S$. aristidis avec respectivement 23/103 et 152/1174 individus jeunes/total, une zone d'occurrence (EOO) de 2 et $2623 \mathrm{~km}^{2}$ et une zone d'occupation (AOO) de 12 et $32 \mathrm{Km}^{2}$. Les menaces identifiées sont les carrières, l'escalade sportive et le béton projeté. Un seul site a été retrouvé pour $S$. auriculifolia (absent dans trois autres sites historiques), avec seulement six individus, tous sénescents, une EOO et une AOO de seulement 0 et $4 \mathrm{Km}$ respectivement. Les menaces y sont mal identifiées. Les nouveaux statuts retenus pour les trois espèces sont, respectivement, "En Danger d'Extinction",

\footnotetext{
*Auteur pour correspondance : melilia.mesbah@gmail.com
} 
«Vulnérable » et «En Danger Critique d'Extinction ». Des mesures de conservation in situ sont très recommandées concernant $S$. sessionis et $S$. aristidis, et une conservation ex situ de $S$. auriculifolia apparaît urgente. L'enjeu patrimonial de ces falaises en Algérie, souvent situées à proximité de villes et/ou d'infrastructures de transport, mérite un renfort des mesures réglementaires (protection légale des espèces, interdiction de techniques d'aménagement) et des mesures de prévention (études d'impact avant projet).

Mots clés : Evaluation UICN, falaises calcaires, menaces, Silene sessionis, Silene aristidis, Silene auriculifolia.

\section{Abstract}

The Mediterranean low-altitude limestone cliffs are known for their exceptional diversity and richness in endemics, often insufficiently assessed in Algeria. The present study is an analysis and a synthesis of individual IUCN assessments of three endemic Algerian chasmophytic species, Silene sessionis, $S$. aristidis and $S$. auriculifolia. The aim is to understand the various threats to this kind of habitat and the conservation issues of the species that grow on it. These assessments were carried out in five littoral or sublittoral areas in the north of the country. Their conservation status was established according to the IUCN recommendations and approved by the official Red List process. New sites were discovered for $S$. sessionis and $S$. aristidis with, respectively, 23/103 and 152/1174 young/total individuals, an extent of occurrence (EOO) / area of occupancy (AOO) of 2/12 and $2.6 / 32 \mathrm{~km}^{2}$. The identified threats are quarries, sport climbing and shotcrete. A single site was found for $S$. auriculifolia (absent in three other historical sites) with only six individuals, all senescent and sterile, an EOO and an AOO of only 0 and $4 \mathrm{~km}$ respectively. Threats are poorly identified. The new adopted statuses for the three species are, respectively, "Endangered", "Vulnerable" and "Critically Endangered". In situ conservation measures are highly recommended for S. sessionis and S. aristidis; ex situ conservation of $S$. auriculifolia appears urgent. The heritage issues of these cliffs in Algeria, often located close to cities and/or transport infrastructures, deserve a reinforcement of regulatory measures (legal protection of species, ban of some development technologies) and preventive measures (pre-project impact assessments).

Keywords: IUCN assessment, limestone cliffs, threats, Silene sessionis, Silene aristidis, Silene auriculifolia..

\section{Introduction}

Les falaises côtières et continentales abritent beaucoup d'espèces et populations reliques qui sont un patrimoine rare et endémique, tant dans la région méditerranéenne (Davis, 1951 ; Médail et Diadema, 2006) que dans le monde entier (Larson et al., 2000). Celles-ci sont considérées comme des zones climatiques de refuge lors des périodes glaciaires du Paléocène (Quézel, 1985 ; Médail et Myers, 2004 ; Dubrowski, 2011 ; Bonanno, 2013). Une telle flore endémique est parfois restreinte à un seul site (site-restricted endemic, SRE) ou plus largement à un territoire biogéographique limité (restricted range endemic, RRE), selon la classification de Radford et al. (2011). Qu'il s'agisse d'espèces RRE ou SRE, les plantes rupicoles 
( « chasmophytes » sensu Davis 1951) constituent une part non négligeable de ces endémiques restreintes (Bacchetta et al., 2007 ; Panitsa et Kontopanou, 2017).

Des endémiques restreintes peuvent être classées à priori en deux catégories principales et opposées, les paléo-endémiques d'une part et les néo-endémiques (dont les schizo-endémiques) d'autre part (Verlaque et al., 1997). Le concept de paléo-endémisme fait référence à une origine lointaine, une longue régression et/ou une longue évolution différenciée avant d'aboutir au taxon actuel, comme Arenaria provincialis Chater et G.Hallyday, une endémique RRE des éboulis et des lapiaz proches de la mer (Youssef et al., 2011) ou divers Sonchus de la section Pustulati, endémiques RRE ou SRE de falaises sublittorales exposées au nord (Silva et al., 2015). Le concept de néo-endémique, quant à lui, fait référence à une fragmentation d'aire de distribution, avec spéciation écologique à la marge, souvent dans des délais bien plus courts, comme Centaurea corymbosa Pourr. (Fréville et al., 1998) et Bupleurum plantagineum Desf. (Neves et Watson, 2004 ; Saadi et Benali, 2015 ; Akroune et Bourkeb, 2016), deux endémiques SRE des falaises méditerranéennes exposées au nord. Ces deux espèces ont en commun une faible capacité à la compétition et une grande spécialisation de leurs habitats (Lavergne et al., 2004 ; Imbert et al., 2012).

Par ailleurs, le rôle crucial de la topographie dans ces systèmes de falaises, particulièrement en exposition nord (Dobrowski, 2010) et/ou en situation sublittorale, suggère l'existence de microclimats particulièrement stables dans le temps et l'existence de micro-refuges ayant pu permettre la persistance des espèces à moyen ou long terme, c'est à dire depuis le dernier cycle glaciaire, voire les précédents. Garcia et al. (2020) suggèrent « une association entre les habitats des reliques, des endémiques et des espèces à leur limite de distribution, et cela dû à un effet stabilisateur des habitats rocheux sur les températures extrêmes ». Ces mêmes auteurs (Garcia et al., 2020) concluent, sur le fait, que « compte tenu du rôle important des habitats rocheux en tant que points chauds de plantes singulières et uniques, leur caractérisation semble une première étape sensée pour identifier les refuges potentiels dans le contexte du changement climatique ». En effet, plusieurs de ces espèces endémiques restreintes (notamment les SRE) ont été considérées, sur les grandes îles de Méditerranée, parmi les espèces les plus en danger, avec des besoins urgents de conservation (Montmollin et Strahm, 2005). L'évaluation de l'état de la biodiversité est un facteur déterminant des priorités de conservation (Bacchetta, 2012). Des listes d'espèces végétales menacées sont fréquemment établies sur la base d'index d'espèces endémiques, rarement générées dans des études antérieures, et en considérant des cas d'espèces en déclin en raison de leur exploitation ou de la destruction de leurs habitats (Garcia et al., 2002).

En Algérie, 39 zones importantes pour les plantes (ZIP) ont été sélectionnées (Yahi et al., 2012 ; Benhouhou et al., 2018). Elles regroupent les habitats les plus riches en endémiques, principalement les montagnes, les îles, les roches sédimentaires calcaires ou dolomitiques et les dunes côtières (Véla et Benhouhou, 2007). Toutefois, l'évaluation des espèces de ces ZIPs repose toujours sur les flores anciennes de Quézel et Santa (1962-1963) ou celle de Maire (1957-1987) datant de la première moitié du siècle mais éditée entre 1952 et 1987. Sur la base de ces flores, les taxa endémiques et rares ont été intégrés dans la liste rouge de l'UICN en 1997 
(Walter et Gillett, 1998). Aucune donnée quantitative ancienne n'a été enregistrée sur cette diversité exceptionnelle. De nombreux travaux partiels, consistant en de simples inventaires floristiques, soulignent le manque d'exploration des données de terrain (Boulemtafes et al., 2018 ; Khennouf et al., 2018 ; Mansouri et al., 2018). Ce manque de données quantitatives ou biogéographiques récentes sur la diversité floristique en Afrique du nord illustre clairement les difficultés à estimer l'état de la biodiversité, sa phylogéographie et sa biogéographie (Médail et Diadema, 2009).

Ces dernières années, l'actualisation in situ des connaissances biogéographiques des espèces endémiques en Algérie, permettant de réviser leurs statuts de conservation et leurs évaluations selon les critères de 1'UICN, a été entamée par Véla et al. (2019) et Mesbah et al. (2019, 2020a, 2020b).

Avec 70 taxa (Dobignard et Chatelain, 2011) dont 25 endémiques, et 15 rares, le genre Silene est celui qui présente la plus forte valeur patrimoniale dans la flore algérienne (Quézel, 1957). Quatre espèces viennent d'être évaluées selon la démarche recommandée par l'UICN (2001) : Silene sessionis Batt. (Mesbah et al., 2019). Silene aristidis Pomel (Mesbah et al., 2020a), Silene auriculifolia Pomel (Mesbah et al., 2020b) et Silene rosulata Soy.-Will. et Gordr. Subsp. rosulata (unpubl. res.). Au regard des chiffres avancés, le genre Silene est désormais le mieux évalué à l'échelle nationale concernant les plantes rupicoles et/ou chasmophytes. C'est pourquoi nous proposons d'utiliser les données récentes produites pour évaluer la vulnérabilité réelle de ces trois espèces. En effet, la connaissance de la répartition de ces espèces était très approximative et ce en raison des données chorologiques anciennes qui étaient peu exploitables. Par conséquent, bien que nous puissions avoir une idée sur leur degré de rareté, nous ne pouvions pas tirer de conclusions sur leurs répartitions réelles.

Dans ce présent travail, nous allons précisément :

(i) analyser la répartition de trois des quatre espèces mentionnées ci-dessus en examinant les zones connues (données anciennes), en confirmant les populations actuelles (nouvelles prospections), en notant éventuellement de nouvelles stations et en documentant les extensions ou extinctions locales ;

(ii) utiliser les données précises sur la taille des populations pour décrire les structures des populations afin de détecter d'éventuelles répartitions déséquilibrées des stades de vie ;

(iii) synthétiser les principales menaces avérées ou potentielles et les possibilités et contraintes pour tenter de les juguler afin de conserver durablement les chasmophytes en général ainsi que leur habitat.

\section{Matériel et méthodes}

\subsection{Zone d'étude}

L'étude a été menée au niveau de deux sites littoraux et trois sites continentaux situés au nord de l'Algérie (Fig.1). Quelques stations ont été ciblées en suivant la distribution ancienne citée dans la bibliographie ou dans les parts d'herbiers. D'autres stations ont été visitées en raison de leur potentiel écologique (falaises calcaires abruptes sur différentes expositions) (Tab. 1). Faute 
de données climatologiques spécifiques aux sites étudiés, nous avons eu recours à la méthode des extrapolations (Seltzer et Auberty, 1946) pour déterminer le type de climat de chacun des sites.

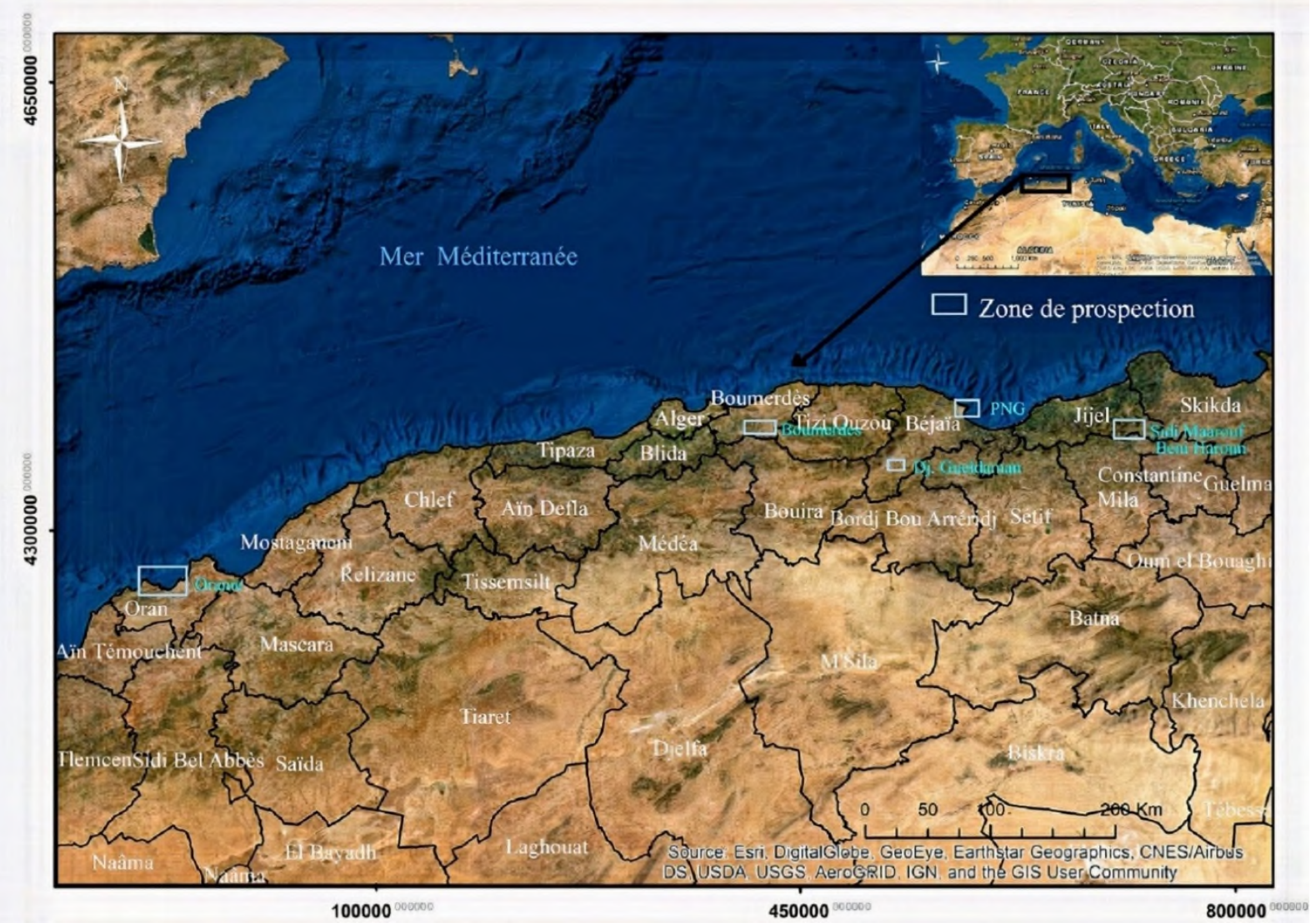

Figure 1 : Situation géographique des zones de prospection.

\subsubsection{Le Parc National du Gouraya (PNG)}

Cette zone d'étude englobe toutes les falaises maritimes accessibles du Parc National de Gouraya en allant du Cap Bouak à l'Est jusqu'à M'cid El Bab à l'Ouest. Cette partie du domaine tellien est d'origine jurassique et est essentiellement constituée de formations calcaires et dolomitiques (Hallal et al., 2018) avec une altitude qui varie du niveau de la mer jusqu'au pic de Yemma Gouraya (660 m d'altitude). Elle appartient au secteur biogéographique de la petite Kabylie ("K2" sensu Quézel et Santa 1962-1963). Elle se caractérise par un bioclimat subhumide à hiver chaud dans sa partie basse, et humide à hiver tempéré pour les hauteurs de YemmaYamna et du Mont Gouraya.

\subsubsection{Les falaises de l'Oranie}

C'est une zone littorale de l'Ouest algérien sur les hauteurs d'El Marsa El Kebir. Les falaises prospectées se situent sur le long du Mont Murdjadjo depuis le fort de Santa Cruz jusqu'aux dernières falaises du mont à $350 \mathrm{~m}$ et $510 \mathrm{~m}$ d'altitude respectivement. À l'Est du Djebel Murdjadjo et à $11 \mathrm{~km}$ de la ville d'Oran, les formations rocheuses du parcours routier suivant 
la piste côtière qui mène de la montagne des lions jusqu'à la ville de Gdyel en passant par Kristel ont été sillonnées. Cette station appartient au secteur biogéographique des collines et du littoral oranais ("O1" sensu Quézel et Santa 1962-1963) caractérisé par un étage bioclimatique semi-aride à hiver chaud.

\subsubsection{Les falaises de la région de Boumerdès}

La prospection a été effectuée sur tous les affleurements rocheux de la chaîne calcaire d'origine liasique et jurassique qui lie le Djebel Bouzegza (Keddara) aux Gorges d'Ammel (anciennement appelées les Gorges de Palestro). Ces falaises sont situées près de la route nationale numéro 5 entre les frontières de la wilaya de Bouira et de Boumerdès en passant par Ighil Waswane, Djerrah et Hini. Ces falaises ont été suivies également sur la rive est de Oued Isser jusqu'à Azrou Imadiyen aux environs de Tala Madi. Ce territoire du secteur biogéographique des collines et du littoral algérois ("A2" sensu Quézel et Santa 1962-1963) présente une variation altitudinale allant de $185 \mathrm{~m}$ aux Gorges de Ammel jusqu'au Sommet de Djebel Bouzegza à $1030 \mathrm{~m}$ d'altitude. Les Gorges d'Ammel jouissent d'un bioclimat subhumide à hiver chaud, alors que les stations d'Ifri N Ziri, Djebel Bouzegza et Azrou Imadiyen se situent dans le bioclimat subhumide à hiver tempéré en raison de leur altitude plus élevée.

\subsubsection{Les falaises de Sidi Maarouf et de Beni Haroun}

Elles sont situées à $50 \mathrm{~km}$ au sud-est de la ville de Jijel vers les frontières avec la wilaya de Mila. Nous les avons parcourues le long de l'Oued El Kebir en passant par les falaises des Hammamet et puis les Gorges de Bin El Ghedrin jusqu'aux Gorges de Beni Haroun qui sont considérées comme les dernières falaises de ce Massif avec une altitude maximale de $360 \mathrm{~m}$. Sur le versant ouest de l'oued, nous avons traversé le monticule rocheux de Ouled Yehya Khedrouche (appelé localement Djebel Zoulem) jusqu'au Mont de Sasnan (respectivement de 280 et $360 \mathrm{~m}$ d'altitude). Une autre formation rocheuse très accidentée sur les hauteurs de Djebel Mantaia à $1190 \mathrm{~m}$ d'altitude a été également visitée. La zone parcourue est située dans le secteur biogéographique des collines du Tell constantinois ("C1" sensu Quézel et Santa 19621963). Les stations de la localité d'El Milia à Jijel se caractérisent par un bioclimat subhumide tempéré pour Bin El Ghedrane, Ouled Yahia Khedrouche, Beni Haroun et un bioclimat humide tempéré pour Sasnan et Ouled Rabah.

\subsubsection{Djebel Gueldaman}

C'est un massif calcaire d'origine liasique du jurassique inférieur du secteur biogéographique des collines du Tell constantinois (C1). Situé à l'est de Béjaïa, il est considéré comme étant la limite occidentale de la chaîne tellienne des Babors. Il est délimité par Oued Soummam sur son versant nord-ouest face à la ville d'Akbou, il est moins accentué que le versant sud. Les altitudes de la ligne de crête sont comprises entre $556 \mathrm{~m}$ et $974 \mathrm{~m}$. Sur le versant sud-est se localise la grotte de Gueldaman d'Akbou (Chaïd-Saoudi, 1987 ; Kherbouche, 2015). Ce massif est soumis à un bioclimat semi-aride à hiver tempéré. 
Bulletin de la Société Royale des Sciences de Liège, Vol. 90, Articles, 2021, p.101 - 124

Tableau 1 : Liste des localités prospectées. SB: Secteur biogéographique.

\begin{tabular}{|c|c|c|c|c|}
\hline Localité & SB & Coordonnées & Altitude (m) & Bibliographie \\
\hline Gorges de Ammel & A1 & $36.611068^{\circ} \mathrm{N}, 3.582852^{\circ} \mathrm{E}$ & 185 & (Pomel, 1875) \\
\hline Ifri N Ziri & A1 & $36.6060025^{\circ} \mathrm{N}, 3.5628702^{\circ} \mathrm{E}$ & 600 & Présent travail \\
\hline Djebal Bouzegza & A1 & $36.605361^{\circ} \mathrm{N}, 3.472250^{\circ} \mathrm{E}$ & 675 & (Pomel, 1875) \\
\hline Gueldamen & $\mathrm{C} 1$ & $36.447866^{\circ} \mathrm{N}, 4.574666^{\circ} \mathrm{E}$ & 500 & $\begin{array}{l}\text { (Véla, Véla-Ouzani et } \\
\text { Rebbas, in prep.) }\end{array}$ \\
\hline Azrou Imaadiyen & A1 & $36.611267^{\circ} \mathrm{N}, 3.613318^{\circ} \mathrm{E}$ & 545 & Présent travail \\
\hline Bin El Ghedran & $\mathrm{K} 2$ & $36.65582^{\circ} \mathrm{N}, 6.2779670^{\circ} \mathrm{E}$ & 200 & $\begin{array}{l}\text { (Jeanmonod, 1982) } \\
\text { (Julien, inédit*) }\end{array}$ \\
\hline $\begin{array}{l}\text { Ouled Yahia } \\
\text { Khedrouche }\end{array}$ & $\mathrm{K} 2$ & $36.658538^{\circ} \mathrm{N}, 6.269895^{\circ} \mathrm{E}$ & 280 & Présent travail \\
\hline Sasnnan & $\mathrm{K} 2$ & $36.657993^{\circ} \mathrm{N}, 6.262043^{\circ} \mathrm{E}$ & 360 & Présent travail \\
\hline Cap Bouak & $\mathrm{K} 2$ & $36.7602786807^{\circ} \mathrm{N}, 5.10538^{\circ} \mathrm{E}$ & 34 & (Battandier, 1914) \\
\hline Cap Noir & $\mathrm{K} 2$ & $36.7692984^{\circ} \mathrm{N}, 5.106657^{\circ} \mathrm{E}$ & 85 & (Battandier, 1914) \\
\hline $\begin{array}{l}\text { Falaise des } \\
\text { Salines }\end{array}$ & $\mathrm{K} 2$ & $36.77134102^{\circ} \mathrm{N}, 5.09382^{\circ} \mathrm{E}$ & 252 & Présent travail \\
\hline YemmaYamna & $\mathrm{K} 2$ & $36.7744468^{\circ} \mathrm{N}, 5.072992^{\circ} \mathrm{E}$ & $501-534$ & Présent travail \\
\hline Djebel Murdjadjo & $\mathrm{O} 1$ & $35.708989^{\circ} \mathrm{N}, 0.671980^{\circ} \mathrm{E}$ & 410 & $\begin{array}{l}\text { (Debeaux \& Dautez, } \\
1889 \text { ) }\end{array}$ \\
\hline Kristel & $\mathrm{O} 1$ & $35.832099^{\circ} \mathrm{N}, 0.472377^{\circ} \mathrm{W}$ & 60 & (Daumas et al. 1952) \\
\hline Djebel Santa Cruz & $\mathrm{O} 1$ & $35.709143^{\circ} \mathrm{N}, 0.666019^{\circ} \mathrm{W}$ & 280 & (Pomel, 1875) \\
\hline $\begin{array}{l}\text { Montagne des } \\
\text { lions }\end{array}$ & $\mathrm{O} 1$ & $35.768720^{\circ} \mathrm{N}, 0.501804^{\circ} \mathrm{W}$ & 545 & Présent travail \\
\hline
\end{tabular}

\subsection{Prospection et dénombrement}

L'exploration des différents sites a eu lieu sur une durée de trois ans (2017-2019). Un échantillonnage ciblé a été effectué sur la base des données bibliographiques et des collections d'herbiers anciens. Cette première orientation des sites potentiels a été complétée par un échantillonnage aléatoire stratifié où d'autres falaises calcaires des régions considérées ont été échantillonnées. Plusieurs sorties de prospection ont été effectuées, du mois de mars au mois de novembre de chaque année, sur des parcours accessibles et à l'aide de jumelles dans les endroits les plus escarpés. Le comptage des individus jeunes et des individus matures est l'opération la plus critique par crainte de confusion entre les catégories d'âge et par le fait que, quelle que soit la précision du comptage, une marge d'erreur est toujours possible. Nous avons considéré comme individus matures toutes les touffes composées de deux ou plusieurs rosettes basales, avec une tige florale ou présence de traces de la floraison de l'année précédente. Toutes les rosettes individuelles composées seulement de feuilles basales sont considérées comme des 
jeunes individus immatures. L'âge approximatif des individus a été déterminé sur la base du nombre de ramifications et du diamètre des tiges (Jeanmonod, 1982).

\section{3. Évaluation du statut de conservation}

Les espèces ont été évaluées sur la base des critères de la liste rouge de l'Union Internationale pour la Conservation de la Nature version 3.1 (UICN 2001). La zone d'occurrence (EOO) et la zone d'occupation (AOO) ont été calculées à l'aide de la plateforme en ligne GeoCat (Bachman, 2011). D'autres données ont été prises en considération telles que le déclin de la population, l'estimation du temps de génération selon la taille des touffes et le nombre de ramifications en floraison et les menaces observées ainsi que leur impact sur la distribution des individus. Les détails de chaque évaluation ont été saisis grâce au Species Information Service (SIS) Toolkit, rev. 2.0., de l'UICN. Chaque évaluation a enfin été validée par les évaluateurs internes de la Liste Rouge de l'UICN avant publication officielle (Mesbah et al., 2019, 2020a, 2020b).

\section{Résultats}

\subsection{Nouvelle distribution connue des espèces du genre Silene}

Silene sessionis a été repérée dans six localités, dont quatre sont considérées comme « nouvelles » par rapport aux connaissances historiques, situées sur les falaises des Salines et les falaises de Yemma Yamna (Fig. 2). Une station de présence potentielle située au-dessous de la carrière de M'cid El Bab demeure non étudiée, car inaccessible et dangereuse à explorer.

En plus du locus classicus (Djebel Bouzegza, Gorges de Palestro et Gorges d'E1 Milia), Silene aristidis a été découverte dans quatre nouvelles stations avec une nouvelle sous-population inédite sur le versant sud du Mont Gueldaman (Akbou) dans la wilaya de Béjaïa. Parmi les stations visitées, $S$. aristidis n'a pas été trouvée sur les rochers des Hammamet et les falaises de Beni Haroun malgré la ressemblance de leur écologie avec celle des sites où elle est présente (Fig. 3). Cependant, le Mont de Mantaya semble ne pas avoir les conditions écologiques favorables à l'installation de cette espèce (haute altitude avec des températures plus basses). Il abrite par contre $S$. andryalifolia Pomel (une ibéro-maghrébine répandue non étudiée ici) que nous avons observée communément dans les falaises de haute altitude telles que dans le mont de Tlemcen, du Djurdjura et des Aurès.

Silene auriculifolia est connue sur le long de la chaîne de Djebel Murdjadjo. Elle a été recherchée et retrouvée aux gorges de Djebel Santo par Jeanmonod (1982) sur le versant ouest de cette chaîne. Nous ne l'avons pas retrouvée lors de notre campagne car cette localité est très anthropisée. Son aire de distribution a été réduite aux derniers faciès rocheux de cette chaîne où nous l'avons repérée péniblement. Nous avons aussi confirmé son absence sur les escarpements de Kristel où elle a été pourtant déjà signalée par Daumas et al. (1952). La Montagne des Lions a été prospectée à cause de la réunion des conditions favorables pour son installation. Notre visite nous a permis de valider son absence dans cette station (Fig. 4). 
Bulletin de la Société Royale des Sciences de Liège, Vol. 90, Articles, 2021, p.101 - 124

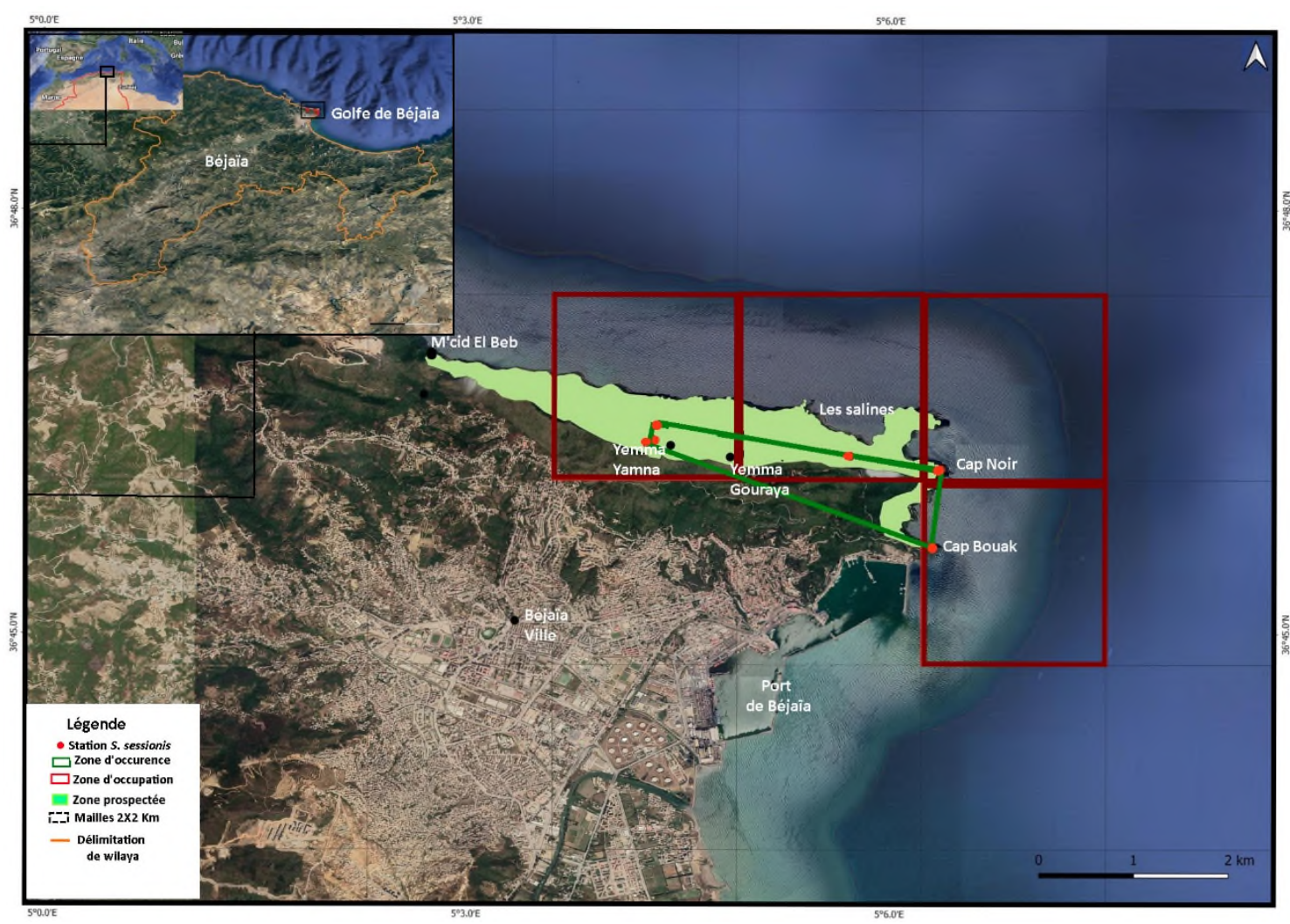

Figure 2 : Répartition actuelle connue de Silene sessionis.

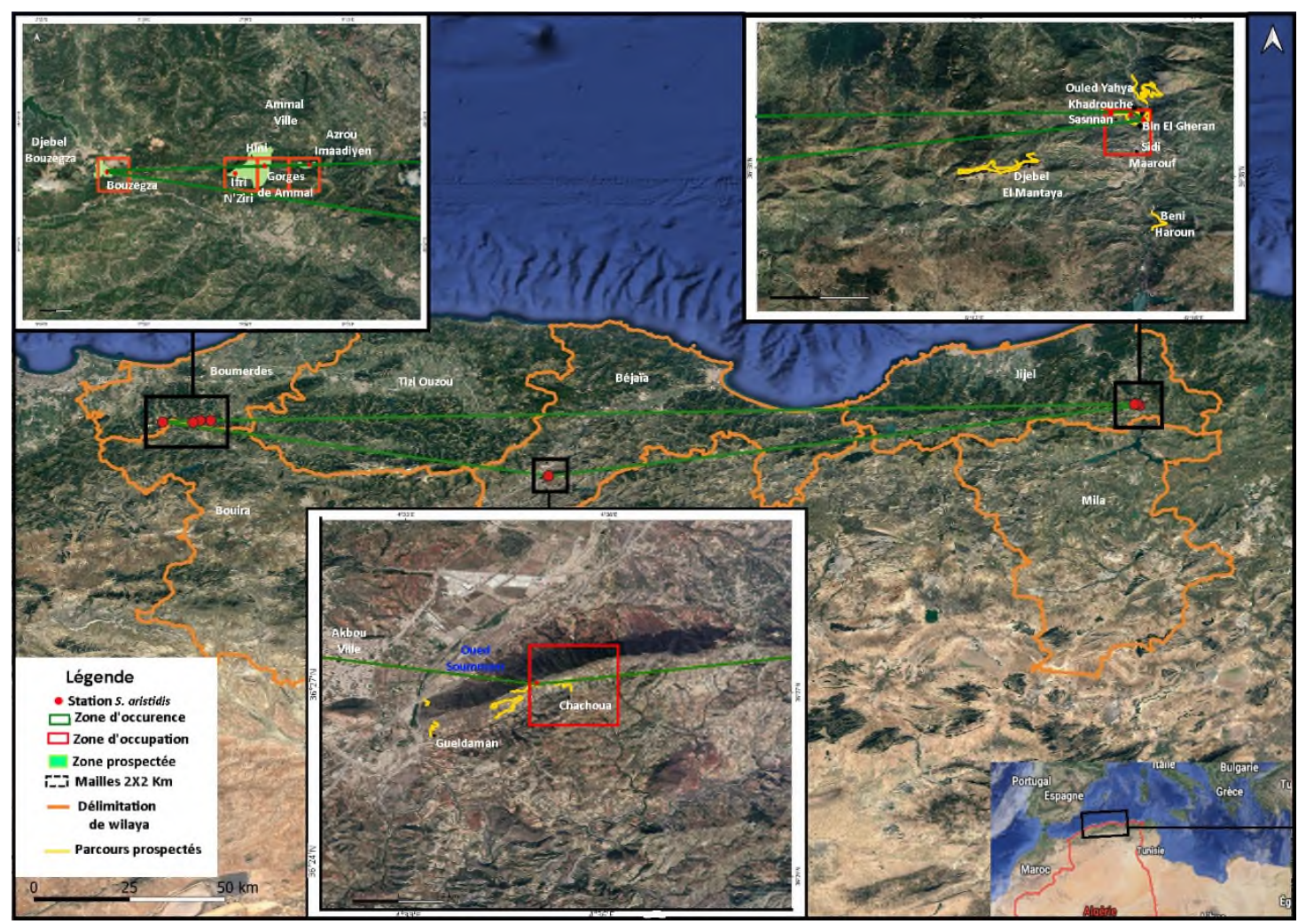

Figure 3 : Répartition actuelle connue de Silene aristidis. 


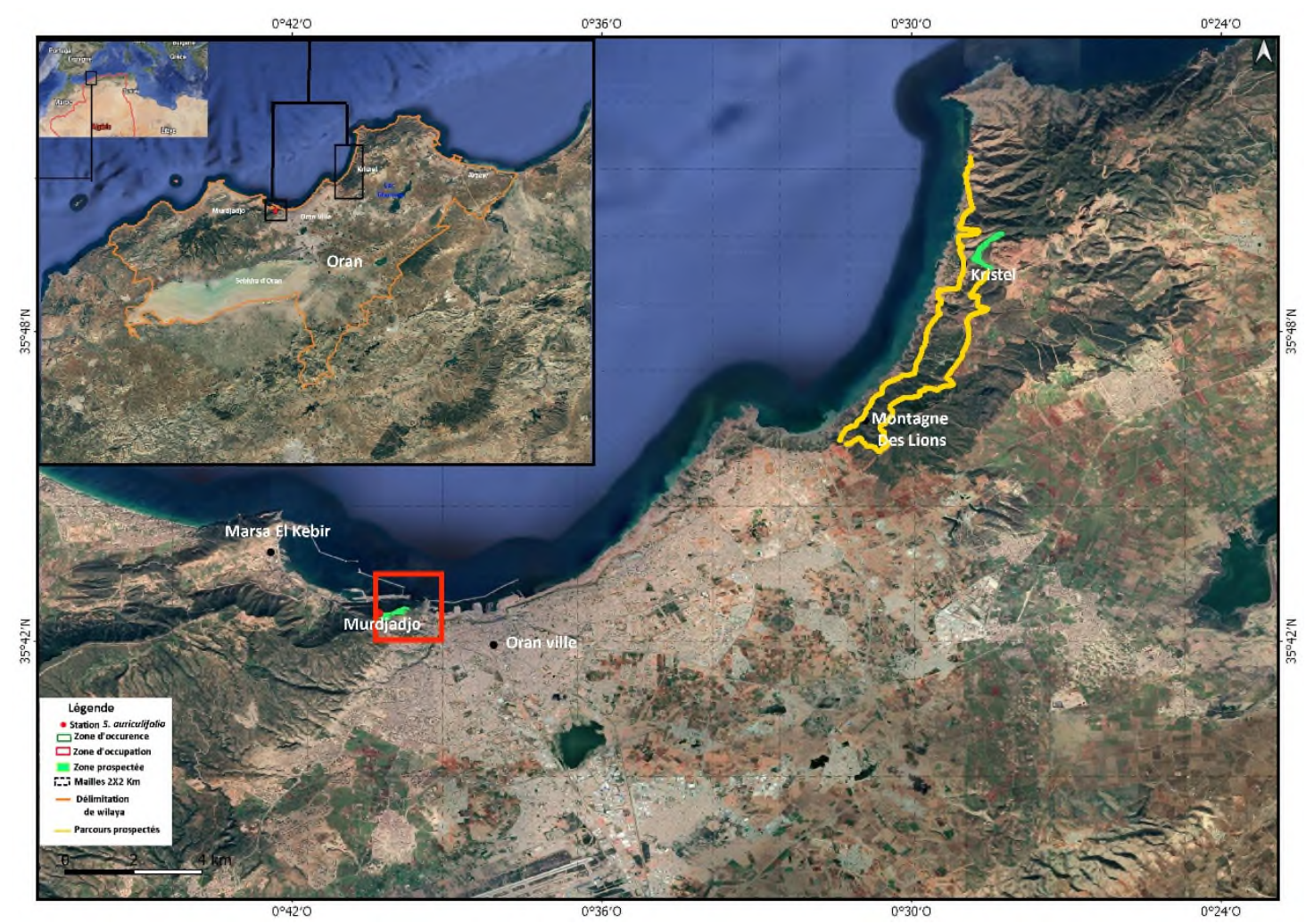

Figure 4 : Répartition actuelle connue de Silene auriculifolia.

\section{2. Évaluation de l'état des populations}

Avant nos travaux (Mesbah et al., 2019, 2020a, 2020b), aucune donnée quantitative de détail n'était disponible pour les trois espèces étudiées. De ce fait, il nous a été impossible d'estimer l'évolution de leurs populations respectives. Avec le détail apporté dans le cadre de cette présente étude, il sera désormais possible de faire un suivi ultérieurement.

Silene aristidis est l'espèce la plus commune avec une large répartition sur trois souspopulations distantes de plus de $40 \mathrm{~km}$. S. sessionis et $S$. auriculifolia sont chacune strictement localisées dans une seule sous-population dont les différentes stations sont rapprochées sans être totalement isolées par les reliefs (Tab. 2).

Les plus grands nombres d'individus matures pour chacun des trois taxa ont été observés dans les stations les plus ombragées qui ne reçoivent que très peu de rayonnements solaires. C'est le cas des falaises du Cap Bouak et Yemma Yamna pour S. sessionis, et Ifri N Ziri pour S. aristidis (Tab. 2).

Le temps de génération n'est pas connu à cause du manque total de données anciennes (cartes de distribution, inventaires des populations, suivis temporels) ou expérimentales (cultures ex situ...). Nous avons estimé, selon la taille des touffes, leur âge à environ 40 à 65 ans en moyenne dans toutes les populations. Ces taxa forment de nombreuses touffes à appareil végétatif bien développé avec une ou plusieurs rosettes basales. L'appareil reproducteur est très variable d'une 
station à une autre et d'un individu à un autre. Il est généralement représenté par une inflorescence très condensée mais quelques-unes seulement atteignent la maturité.

Sur la base de nos observations, la forme sénescente est repérable par des touffes qui ont un aspect maigre dont la plupart des rameaux sont stériles ou à fleurs avortées avant maturité. C'est le cas des individus observés dans la population de $S$. auriculifolia.

L'absence d'individus jeunes chez $S$. auriculifolia et/ou la présence d'un nombre réduit d'individus jeunes chez $S$. sessionis (Tab. 2) implique une faible probabilité de succès de la régénération naturelle. Il est encore tôt pour considérer ces espèces comme étant en difficulté de régénération, étant donné que leur espérance de vie est longue et que les individus atteignent la maturité à partir de la deuxième ou la troisième année, sauf dans le cas de $S$. auriculifolia qui présente des individus sénescents et aucun jeune sujet pour assurer la régénération. Silene aristidis présente un équilibre entre le nombre d'individus jeunes et celui d'individus matures. L'observation d'individus de différentes générations chez ce dernier indique sa bonne régénération naturelle.

Tableau 2 : Distribution des individus jeunes et des individus matures dans les différentes populations des trois espèces étudiées. N. = Nombre d'individus. *Échantillon d'herbier déposé à MNHN (Numéro d'inventaire : P05160214)

\begin{tabular}{|c|c|c|c|c|c|}
\hline Espèce & Localité & $\begin{array}{c}\text { Altitude } \\
(\mathrm{m})\end{array}$ & Exposition & $\begin{array}{l}\mathrm{N} . \\
\text { total }\end{array}$ & N. jeunes \\
\hline \multirow{8}{*}{ Silene sessionis } & Cap Bouak & 34 & Nord & 40 & $7-10$ \\
\hline & Cap Noir & 85 & Nord & 1 & 0 \\
\hline & Falaises des Salines & 252 & Nord-est & 12 & 2 \\
\hline & YemmaYamna Nord & 501 & Nord & 46 & $7-10$ \\
\hline & $\begin{array}{l}\text { Falaises au-delà } \\
\text { deYemmaYamna en venant } \\
\text { de Yemma Gouraya }\end{array}$ & 534 & Nord-ouest & 4 & 1 \\
\hline & $\begin{array}{l}\text { Falaises en bas de Yemma } \\
\text { Yemma }\end{array}$ & 490 & Nord & 3 & 0 \\
\hline & \multirow{2}{*}{\multicolumn{3}{|c|}{ Total : }} & 103 & 23 \\
\hline & & & & $10 J$ & $22.33 \%$ \\
\hline \multirow{10}{*}{ Silene aristidis } & Gorges de Ammel & 185 & Nord-est & 214 & 15 \\
\hline & Ifri n’ Ziri & 600 & Nord & 598 & 90 \\
\hline & Djebel Bouzegza & 675 & Nord & 89 & 20 \\
\hline & Azrou Imaadiyen & 545 & Nord-est & 109 & 10 \\
\hline & Bin El Ghedran & 200 & Nord-ouest & 16 & 0 \\
\hline & Ouled Yahia Khedrouche & 280 & Nord est & 47 & 7 \\
\hline & Sasnnan & 360 & Nord & 51 & 10 \\
\hline & Gueldaman & 500 & Sud-est & 50 & 0 \\
\hline & \multirow{2}{*}{\multicolumn{3}{|c|}{ Total : }} & 117 & 152 \\
\hline & & & & 4 & $12,95 \%$ \\
\hline Silene auriculifolia & Djebel Murdjadjo & 410 & Nord & 6 & 0 \\
\hline
\end{tabular}




\section{3. Évaluation du statut UICN}

D'après les données de terrain et la cartographie des populations (Tab. 3), l'application de la charte des critères de l'UICN (Mesbah et al., 2019, 2020a, 2020b) est rappelée ci-dessous.

Dans le cas de Silene sessionis, les critères A et E de la charte UICN ne sont pas applicables en raison du manque de données. De plus, les critères $\mathrm{B}$ et $\mathrm{C}$ ne permettent pas d'atteindre les seuils de menace, car pas de déclin connu ni supposé ou prévu. Dans notre cas, seul le critère D est applicable avec 95 individus matures, 6 localités et $4 \mathrm{~km}^{2}$ de zone d'occupation (Fig. 2). Ainsi, le statut à accorder à cette espèce est celui « En Danger d'Extinction » (EN) selon le critère D1. La principale menace pesant sur cette espèce est l'aménagement de pistes d'escalades au sein de son habitat.

Silene aristidis possède une population de 1024 individus matures, 8 sous-populations, une zone d'occurrence (EOO) estimée à $2623 \mathrm{~km}^{2}$ et une zone d'occupation (AOO) de $32 \mathrm{~km}^{2}$. Celle-ci est donc évaluée comme « Vulnérable » (VU : A3c; B1ab (iii,iv,v) + 2ab (iii,iv,v); C2a (i)) en raison de sa population naturelle restreinte et du probable déclin futur de la population du fait de la présence de certaines menaces actives telles que les carrières proches des localités de Bouzegza et Ifri N Ziri, et le béton projeté sur les falaises des Gorges d'Ammel et de Bin El Ghedrin. Le critère E n'a pas été appliqué par manque de données. Le critère D2 n'a pas été retenu car l'AOO est supérieure à $20 \mathrm{~km}^{2}$ et le nombre de localités est très supérieur à 5 . Le critère D1 est très proche du seuil de 1000 .

Silene auriculifolia est classée dans la catégorie « En Danger Critique d'Extinction » (CR: $\mathrm{B} 1 \mathrm{ab}(\mathrm{iv}, \mathrm{v})+2 \mathrm{ab}(\mathrm{iv}, \mathrm{v})$; $\mathrm{C} 2 \mathrm{a}(\mathrm{i}, \mathrm{ii})$; D) en raison de sa très faible population (moins de 50 individus matures) qui est présumée être en déclin. À ce stade, les menaces connues sont très peu nombreuses, telles que l'accessibilité de son habitat naturel in situ et sa difficulté à se régénérer. La principale cause du déclin de la population reste problématique. Les critères A et E ne sont pas applicables dans le cas de cette espèce par manque de données. 
Tableau 3 : Résultats de l'évaluation des espèces de Silene sect. Siphonomorpha selon les critères de l'UICN version 3.1 (Mesbah et al., 2019, 2020a, 2020b) et les critères d'avant 1994 (Walter \& Gillet 1998).Sp1 : Silene sessionis ;Sp2 : Silene arisistidis; Sp3 : Silene auriculifolia; NIM: Nombre d'individus matures; Dm : Données manquantes ; E: Endémique; R: Rare; EN: En danger d'Extinction; VU: Vulnérable; CR: En danger critique d'Extinction.

\begin{tabular}{|c|c|c|c|c|c|}
\hline Cat & \multicolumn{2}{|l|}{ Indice de classification } & Sp1 & Sp2 & Sp3 \\
\hline \multicolumn{6}{|c|}{$\begin{array}{c}\text { A. Réduction de la taille de la population. } \\
\text { Réduction de la population constatée, estimée, déduite ou supposée, }\end{array}$} \\
\hline A1 & $\begin{array}{l}\text { Dans le passé, lorsque les causes de la } \\
\text { réduction sont clairement } \\
\text { réversibles ET comprises ET ont cessé. ( } \geq \\
90 \%=\mathrm{CR}, \geq 70 \%=\mathrm{EN}, \geq 50 \%=\mathrm{VU} \text { ) }\end{array}$ & \multirow{4}{*}{$\begin{array}{l}\text { Les critères A1,2,3 et } 4 \text { sont pris en compte } \\
\text { en se basant sur l'un des éléments suivants: } \\
\text { (a) l'observation directe [excepté A3] (b) } \\
\text { un indice d'abondance adapté au taxon (c) } \\
\text { la réduction de la zone d'occupation } \\
\text { (AOO), de la zone d'occurrence (EOO) } \\
\text { et/ou de la qualité de l'habitat (d) les } \\
\text { niveaux d'exploitation réels ou potentiels } \\
\text { (e) les effets de taxons introduits, de } \\
\text { l'hybridation, d'agents pathogènes, de } \\
\text { substances polluantes, d'espèces } \\
\text { concurrentes ou parasites }\end{array}$} & $\mathrm{Dm}$ & Non & $\mathrm{Dm}$ \\
\hline A2 & $\begin{array}{l}\text { Dans le passé, lorsque les causes de la } \\
\text { réduction n'ont peut-être pas cessé OU ne } \\
\text { sont peut-être pas comprises OU ne sont peut- } \\
\text { être pas réversibles }(\geq 80 \%=\mathrm{CR}, \geq 50 \%=\mathrm{EN}, \geq \\
30 \%=\mathrm{VU})\end{array}$ & & $\mathrm{Dm}$ & Non & $\mathrm{Dm}$ \\
\hline A3 & $\begin{array}{l}\text { Dans le futur (sur un max. } 100 \text { ans) } \\
(\geq 80 \%=\mathrm{CR}, \geq 50 \%=\mathrm{EN}, \geq 30 \%=\mathrm{VU})\end{array}$ & & $\mathrm{Dm}$ & Oui & $\mathrm{Dm}$ \\
\hline A4 & $\begin{array}{l}\text { Sur une période de temps devant inclure à la } \\
\text { fois le passé et l'avenir (sur un max. } 100 \text { ans } \\
\text { dans le futur), lorsque les causes de la } \\
\text { réduction n'ont peut-être pas cessé OU ne } \\
\text { sont peut-être pas comprises OU ne sont peut- } \\
\text { être pas réversibles. ( } \geq 80 \%=C R, \geq 50 \%=E N, \geq \\
30 \%=V U)\end{array}$ & & $\mathrm{Dm}$ & Non & $\mathrm{Dm}$ \\
\hline \multicolumn{6}{|c|}{ B. Répartition géographique, qu'il s'agisse de B1 (zone d'occurrence) ET/OU B2 (zone d'occupation) } \\
\hline B1 & \multicolumn{2}{|c|}{ Zone d'occurrence $(\mathrm{EOO})\left(<100 \mathrm{~km}^{2}=\mathrm{CR},<5000 \mathrm{~km}^{2}=\mathrm{EN},<20000 \mathrm{~km}^{2}=\mathrm{VU}\right)$} & 2 & $\begin{array}{r}262 \\
3\end{array}$ & 0 \\
\hline B2. & \multicolumn{2}{|c|}{ Zone d'occupation (AOO) $\left(<10 \mathrm{~km}^{2}=\mathrm{CR}, \leq 500=\mathrm{EN}, \leq 2000=\mathrm{VU}\right)$} & 12 & 32 & 4 \\
\hline B. n.a & \multicolumn{2}{|c|}{ Sévèrement fragmentée OU nombre de localités $(1=\mathrm{CR}, \leq 5=\mathrm{EN}, \leq 10=\mathrm{VU})$} & Non & Oui & $\begin{array}{l}\text { Oui } \\
\text { B.1.2 }\end{array}$ \\
\hline B.n.b & \multicolumn{2}{|c|}{$\begin{array}{l}\text { Déclin continu constaté, estimé, déduit ou prévu de l'un des éléments suivants : (i) zone } \\
\text { d'occurrence, (ii) zone d'occupation, (iii) superficie, étendue et/ou qualité de l'habitat, (iv) } \\
\text { nombre de localités ou de sous-populations, (v) nombre d'individus matures }\end{array}$} & Non & $\begin{array}{l}\text { Oui } \\
\text { B.1et } \\
\text { 2(iii, } \\
\text { iv, v) }\end{array}$ & $\begin{array}{l}\text { Oui } \\
\text { B.1et } \\
\text { 2(iii, iv, } \\
\text { v) }\end{array}$ \\
\hline B.n.c & \multicolumn{2}{|c|}{$\begin{array}{l}\text { Fluctuations extrêmes de l'un des éléments suivants : (i) zone d'occurrence, (ii) zone } \\
\text { d'occupation, (iii) nombre de localités ou de sous-populations, (iv) nombre d'individus } \\
\text { matures }\end{array}$} & Non & Non & Non \\
\hline \multicolumn{6}{|c|}{$\begin{array}{l}\text { C. Petite population et déclin } \\
\text { NIM }(<\mathbf{2 5 0}=\mathbf{C R}, \leq \mathbf{2 5 0 0}=\mathbf{E N}, \leq \mathbf{1 0} \mathbf{0 0 0}=\mathbf{V U}) \text { ET au moins un des sous-critères } \mathrm{C} 1 \text { ou } \mathrm{C} 2 \text { : }\end{array}$} \\
\hline C1. & \multicolumn{2}{|c|}{$\begin{array}{l}\text { Un déclin continu constaté, estimé ou prévu (sur un max. de } 100 \text { ans dans le futur) }(25 \% \\
\text { en } 3 \text { ans ou } 1 \text { génération=CR, } 20 \% \text { en } 5 \text { ans ou } 2 \text { générations }=\mathrm{EN}, 10 \% \text { en } 10 \text { ans ou } 3 \\
\text { générations }=\mathrm{VU})\end{array}$} & Non & Non & Non \\
\hline C2.a & \multicolumn{2}{|c|}{$\begin{array}{l}\text { Un déclin continu constaté, estimé, prévu ou déduit ET au moins } 1 \text { des } 3 \text { conditions } \\
\text { suivantes : (i) Nombre d'individus matures dans chaque sous-population }=\left(<50 \mathrm{~km}^{2}=\mathrm{CR} \text {, }\right. \\
\left.<250 \mathrm{~km}^{2}=\mathrm{EN},<1000 \mathrm{~km}^{2}=\mathrm{VU}\right) \\
\text { Ou (ii) } \% \text { d'individus matures dans une sous-population }=(90-100 \%=\mathrm{CR}, 95-100 \%=\mathrm{EN} \text {, } \\
<100 \%=\mathrm{VU})\end{array}$} & Non & $\begin{array}{l}\text { Oui } \\
\text { C.2.a } \\
\text { (i) }\end{array}$ & $\begin{array}{l}\text { Oui } \\
\text { C.2.a } \\
(\mathrm{i}, \mathrm{ii})\end{array}$ \\
\hline C2.b & \multicolumn{2}{|c|}{ Fluctuations extrêmes du nombre d'individus matures } & Non & Non & Non \\
\hline \multicolumn{6}{|c|}{ D. Population très petite ou restreinte } \\
\hline D & \multicolumn{2}{|c|}{ Nombre d'individus matures $(<50=\mathrm{CR},<250=\mathrm{EN})$} & 95 & Non & 6 \\
\hline D1 & \multicolumn{2}{|c|}{ Nombre d'individus $<1000=\mathrm{VU}$} & Non & 1022 & Non \\
\hline D2 & \multicolumn{2}{|c|}{ Pour la catégorie VU uniquement : AOO $<20 \mathrm{~km}^{2}$ ou nombre de localités $\leq 5$} & 6 & Non & Non \\
\hline $\mathbf{E}$ & \multicolumn{2}{|l|}{ Probabilité d'extinction dans la nature } & $\mathrm{Dm}$ & $\mathrm{Dm}$ & $\mathrm{Dm}$ \\
\hline & \multicolumn{2}{|l|}{ Catégorie UICN 1998} & $\mathrm{E}$ & $\mathrm{R}$ & / \\
\hline & \multicolumn{2}{|l|}{ Catégorie UICN retenue $\mathbf{2 0 1 9 / 2 0 2 0}$} & EN & VU & $\mathrm{CR}$ \\
\hline
\end{tabular}


Les deux évaluations avant et après 2001 (Walter \& Gillet, 1998 ; Mesbah et al., 2019, 2020a, $2020 b$ ) montrent une stabilité pour Silene sessionis et une aggravation pour S. aristidis. Silene auriculifolia n'avait pas été évaluée, vraisemblablement par négligence des taxa considérés alors à un rang infra-spécifique, comme ce fut le cas pour S. mollissima subsp. auriculifoIia (Pomel) Maire.

\subsection{Les menaces}

Parmi les différentes menaces qui pèsent sur les trois taxa étudiés, nous détaillons ci-dessous les plus préoccupantes, à savoir les carrières, le béton projeté et l'escalade.

\subsubsection{Les carrières}

Silene sessionis est touchée par l'exploitation d'une carrière située au cœur du Parc National de Gouraya. De plus, celle-ci rend l'accès impossible aux falaises calcaires de M'cid El Bab, habitat potentiel d'un bon nombre d'espèces à haute valeur patrimoniale dont $S$. sessionis, dont nous n'avons pu à ce jour confirmer ni la présence ni l'absence

Pour $S$. aristidis, la sous-population de Boumerdès est entourée de cinq carrières plus ou moins proches. La carrière de Bouzegza est celle qui a le plus d'impact sur la survie des espèces rupicoles des falaises et donc des endémiques qu'elles hébergent.

\subsubsection{Le béton projeté}

Les extensions de travaux de protection des murailles des falaises au bord de la route ont été observées près des Gorges de Ammel et les Gorges de Bin El Ghadrin. Ces activités sont régulièrement réalisées dans les zones à forte fréquence d'éboulement (Slimi et Larue, 2015). Les travaux avec le béton projeté constituent indéniablement une forte menace pour les espèces poussant dans les falaises, dont Silene aristidis.

\subsubsection{L'escalade}

Des pistes d'escalade préjudiciables à une végétation rupicole parfois unique ont été aménagées récemment au Cap Carbon à Béjaïa et aux Gorges d'Ammel à Boumerdès.

\section{Discussion}

\section{1. Écologie stationnelle}

Les taxa chasmophytes endémiques de très basse et basse altitude de l'étage bioclimatique subhumide poussent exclusivement sur des falaises calcaires ombragées exposées au nord, nord-est ou nord-ouest, et exceptionnellement sud-ouest ou ouest. La végétation environnante est dominée par les espèces des formations sclérophylles méditerranéennes telles que Olea europaea L. var. oleaster, Ceratonia siliqua L., Pistacia lentiscus L., Quercus coccifera L., etc. Les falaises elles-mêmes sont également caractérisées par la présence de divers taxa rupicoles dont la plupart sont des sténo-endémiques comme Hypochaeris saldensis Batt., Bupleurum plantagineum Desf., Cheiranthus cheiri (L.) Crantz subsp. inexpectans Véla, Ouarmin et 
Dubset dans le cas des falaises de Gouraya, et l'endémique Fumaria mairei Pugsley ex Maire dans le cas des Gorges de Ammel. Quant aux falaises semi-arides de la côte ouest algérienne (Oran), elles sont recouvertes par une végétation éparse dominée par Pinus halepensis Mill., Chamaerops humilis L., Daphne gnidium L. etc. Ces falaises présentent également divers taxa rupicoles comme Campanula mollis L. et Silene pseudoatocion Desf. et quelques espèces rares telles que Caralluma europaea (Guss.) Murb., etc...

Les falaises riches en endémiques en Méditerranée sont caractérisées par une exposition très majoritairement nord, un bioclimat relativement frais, voire humide, et une très forte pente. Ces habitats particuliers sont considérés comme des zones refuges durant les périodes de glaciation favorisant la propagation des endémiques lors des périodes postglaciaires (Tassin, 2012). Les espèces endémiques strictes des falaises calcaires ont tendance à ne pas trop remonter en altitude ni à coloniser les expositions sud.

L'abri des gorges a toujours été considéré comme l'habitat favorable de Silene aristidis (Pomel, 1888; Maire, 1963; Jeanmonod, 1982). D'après nos observations, il semble que ce chasmophyte ait pu trouver refuge lors des périodes glaciaires dans les fissures des rochers calcaires du Lias et du Jurassique, y compris à moyenne altitude. Cette hypothèse ouvre la perspective de retrouver d'autres stations sur la ligne continentale de chaînons calcaires encore mal prospectés, telles les falaises de Beni Ourtilane et de Hammam Guergour (wilaya de Sétif), les falaises de Chabet el-Akra dans les Gorges de Kherrata, ou les falaises d'Ibn Ziyad et les gorges du Rhumel à Constantine. Cela nous encourage également à étendre la recherche des endémiques littorales au niveau des falaises ouest de Gouraya (Djebel Oufarnou), dans les falaises inaccessibles de Cap Aokas et de Melbou (Béjaïa), les Gorges de Dar el-Oued et de Taza à Jijel, etc.

$\mathrm{Au}$ niveau stationnel, le succès de régénération est tributaire des multiples conditions qui doivent être simultanément remplies pour que la germination, l'installation et la croissance des plantes réussissent (Baraloto, 2003). Chez les populations étudiées, l'affaiblissement du pouvoir germinatif des graines ou la mortalité des jeunes plantules sont probablement dus aux facteurs abiotiques (sécheresse estivale) et biotiques (insectes abimant les fleurs et autres animaux broutant le plantules) de l'environnement. La régénération des espèces pérennes est une notion complexe qui doit être estimée sur plusieurs années car le pouvoir germinatif peut varier d'une année à une autre, et nous manquons à ce jour du recul nécessaire.

\subsection{Enjeux de conservation}

$\mathrm{Vu}$ leur accessibilité difficile, les falaises calcaires sont les habitats les plus naturellement conservés, protégés et les moins affectés par l'Homme (Véla et Benhouhou, 2007; Larson et $a l ., 2005)$. Néanmoins, trois types de menaces anthropiques pèsent sur les populations des espèces étudiées : les carrières, le bétonnage des falaises et l'escalade sportive.

Les carrières

L'extraction en carrière est une activité agressive envers la nature et provoque la destruction des habitats et la perturbation de la faune et de la flore (Chenot et al., 2018). Afin de protéger la diversité faunistique et floristique de ces habitats, il est recommandé de sélectionner des 
localités qui ne présentent pas des espèces à haute valeur patrimoniale. Les carrières installées à l'intérieur des aires protégées doivent être fermées et restaurées en respectant la trajectoire historique de l'écosystème d'avant la perturbation. Les carrières abandonnées peuvent devenir, à moyen terme, un refuge aux espèces menacées par manque de fréquentation humaine (ChenotLescure et Lescure, 2019). Les falaises de basses et de moyennes altitudes sont en connectivité permanente avec le développement urbain et l'aménagement routier.

La problématique des carrières parait plus modérée lorsqu'elles s'attaquent à un versant de pente. Celles-ci créent ainsi un front de taille artificiel sur une pente montagneuse de maquis plus ou moins forestier, ce qui n'est pas le type d'habitat le plus riche en endémiques et/ou en espèces rares. Nous pouvons citer en exemple la colline située au sud de la commune d'Akbou (wilaya de Béjaïa), non loin du site de Gueldaman mais qui ne présente pas du tout le même intérêt botanique. En revanche, le problème atteint son paroxysme quand les carrières s'attaquent directement à des fronts de falaises naturelles, hébergeant de nombreuses espèces endémiques et/ou reliques rares. Nous avons en tête un exemple frappant au nord de la commune de M'hir (wilaya de Bordj-Bou-Arreridj), site situé en plein cœur de la ZIP (Zone Importante pour les Plantes) des Bibans (Benhouhou et al., 2018).

Le bétonnage des falaises

La création de passages routiers au bord des falaises est toujours suivie par le risque d'éboulement rocheux (Slimi et Larue, 2015). Pour cela, les décideurs optent de plus en plus pour les aménagements routiers en béton projeté.

Les ions du béton projeté affectent le $\mathrm{pH}$ et la conductivité électrique des eaux consommées par les plantes, ce qui empêche l'installation des espèces rupicoles. Les métaux lourds ajoutés pour augmenter la consistance du béton peuvent être tolérés par les plantes jusqu'à un certain degré (Purdy et Wright, 2019). Notons néanmoins qu'il est possible de rendre le béton projeté viable pour la végétation par l'alcalinisation de sa constituante minérale (Bidou et Guiraud, 2012). Cette étude peut être une piste intéressante à privilégier car permettant d'acquérir des données sur les exigences pédologiques de la flore rupicole et de protéger la biodiversité tout en assurant la protection routière.

D'autres sites potentiellement intéressants pour d'autres espèces à haute valeur patrimoniale telles que les Gorges de Beni Haroun (Véla et al., 2008), les Gorges de la Chiffa ou les Gorges de Taza font face au même problème d'éboulements et donc à la tentation d'y pratiquer la sécurisation par béton projeté. Tous sont aujourd'hui reconnus comme ZIP (Benhouhou et al., 2018) et les deux derniers sont même inclus dans les périmètres respectifs du parc national de Chréa et celui de Taza.

L'escalade sportive

Durant les deux dernières décennies, l'escalade est devenue une activité sportive prisée en Algérie et qui par ailleurs se développe d'une manière exponentielle sur la rive Nord de la Méditerranée (Lorite et al., 2017). Cette pratique est considérée comme un danger potentiel à la présence des endémiques des falaises (Nuzzo, 1995), dans les sites qui en abritent. Elle 
engendre une perte significative du couvert végétal par l'arrachage d'individus ou de tiges florales (Nuzzo, 1995; Schmera et al., 2018), une perte dans la richesse spécifique et une modification de l'écosystème (Lorite et al., 2017).

Dans un futur proche et pour répondre à la demande des passionnés de cette activité, d'autres pistes d'escalade risquent d'être créées si des mises en garde générales, voire des interdictions localisées, ne sont pas exprimées.

Autres menaces potentielles

Un assèchement des falaises a été remarqué dans quelques stations (Falaise des Salines au Parc National de Gouraya, les falaises du Djebel Murdjadjo). L'impact de la fluctuation des précipitations annuelles ou des températures sur le substrat rocheux ne peut être déduit que sur une synthèse climatique de plusieurs années (Soubeyroux et al., 2012). La notion de la sécheresse est très complexe du fait qu'elle est liée à plusieurs paramètres autres que les précipitations et les températures : la durée dans le temps, l'intensité, l'étendue géographique, les exigences de la végétation en humidité... (Wilhite et Glantz, 1985). Une synthèse climatique régionale détaillé serait d'une grande utilité pour se prononcer sur l'impact futur de la sécheresse sur la biodiversité des falaises.

\subsection{Perspectives pour la conservation}

Le nouveau statut retenu pour Silene sessionis, Silene aristidis et Silene auriculifolia selon la version 3.1 (2001) est respectivement celui d'espèce « En Danger d'Extinction (EN) », «Vulnérable (VU) » et « En Danger Critique d'Extinction (CR)» (Mesbah et al., 2019, 2020a, 2020b). Ces trois évaluations justifient amplement la mise en place d'un protocole de suivi régulier dans l'avenir afin d'améliorer les connaissances biologiques et écologiques, in situ et ex situ, de ces espèces et de surveiller l'état de leurs populations. De plus, nous recommandons d'inclure les falaises de Sidi Marouf dans une ZIP élargie basée sur celle de Beni Haroun (Benhouhou et al., 2018).

Dans le cas de Silene auriculifolia, nous avons été confrontés à la difficulté de définir la vraie menace pour pouvoir proposer des actions pour sa conservation. Nous avons estimé sa disparition probable (spontanée ?) dans les prochaines dizaines d'années. Il est donc urgent de prendre des mesures de conservation ex situ par la conservation de graines, une culture in vitro et une tentative de germination dans des jardins botaniques à des fins de renforcement voire de réintroduction future. C'est probablement également le cas de plusieurs autres endémiques de l'Algérie ou d'Afrique du Nord qui sont très mal connues et rarement observées.

Sur le plan législatif, les actions anthropiques (carrières, béton projeté et escalade), qui menacent en priorité les taxa chasmophytes concernés dans cette étude, devraient être soumises à des études d'impact sur la biodiversité, à l'échelle locale et nationale. Pour aider en ce sens, il serait sans doute pertinent et utile d'inclure les espèces Silene aristidis et $S$. auriculifolia dans la liste du « Décret exécutif $\mathrm{n}^{\circ}$ 12-03 du (...) 4 janvier 2012 fixant la liste des espèces végétales non cultivées protégées ». Heureusement, l'inaccessibilité des sites à cause de leurs 
escarpements constitue encore un avantage important de protection naturelle la plupart du temps.

Un diagnostic écologique approfondi des habitats rupicoles passe par la connaissance des facteurs écologiques déterminants sur l'ensemble des espèces de ces milieux, notamment les chasmophytes endémiques dont il est question dans cette étude. Malheureusement, très souvent la mise en place d'une démarche efficace de conservation est limitée par le manque de connaissances biogéographiques, écologiques et biologiques (temps de génération, âge à la maturité, âge de sénescence, taux de germination, etc.) des espèces étudiées (Baccheta et al., 2012). Cependant, les nouvelles technologies permettent de faire des observations inédites comme par exemple le développement de la méthode FPP (Fixed Point Photography) (Anderson et Kevin, 2013) et la technique PPM (Photo Point Monitoring) (Hall, 2002) ou encore l'utilisation des drones pour la photographie aérienne, UAV (Unmanned Aerial Vehicles) (Strumia, 2020).

\section{Remerciements}

Cette étude a été réalisée dans le cadre des activités de recherche du Laboratoire d'Ecologie et environnement (FSNV, Université de Bejaia) affilié à la Direction Générale de la Recherche Scientifique et du développement Technologique (DGRDT), MESRS, Algérie. Nous exprimons notre gratitude aux conservations des forêts notamment celle de Jijel pour leur accompagnement sur le terrain ainsi que toutes les personnes qui nous ont soutenu(e)s par leur disponibilité, leurs conseils et leur apport scientifique. L'exploration botanique du versant sud du Djebel Gueldamane (Véla, Véla Ouzzani \& Rebbas, in prep.) a été encouragée par le Dr Farid Kherbouche du Centre National de Recherche Préhistorique, Anthropologique et Historique (CNRPAH, Alger).

\section{Références}

Akroune L., Bourkeb T., 2016. Distribution et taille de la population de Buplèvre à feuilles de plantain (Bupleurum plantagineum Desf.) dans la zone centrale et occidentale du Parc National du Gouraya (Kabylie des Babors, Algérie). Mémoire de Master en Biologie de la conservation. Béjaïa (Algérie), Université de Bejaia, Algérie. http://www.univbejaia.dz/jspui/handle/123456789/10377

Anderson K., Gaston K.J., 2013. Lightweight unmanned aerial vehicles will revolutionize spatial ecology. Frontiers in Ecology and the Environment, 11(3): 138-146.

https://doi.org/10.1890/120150

Bacchetta G., Casti M., Mossa L., 2007. New ecological and distributive data on the rupestrian flora of Sardinia. Journal de Botanique de la Société Botanique de France, 38 : 73-83.

Bacchetta G., Farris E., Pontecorvo C., 2012. A new method to set conservation priorities in biodiversity hotspots. Plant Biosystems, 146(3): 638648

https://doi.org/10.1080/11263504.2011.642417 
Bulletin de la Société Royale des Sciences de Liège, Vol. 90, Articles, 2021, p.101 - 124

Bachman S., Moat J., Hill A., de la Torre J., Scott B., 2011. Supporting Red List threat assessments with GeoCAT: Geospatial conservation assessment tool. ZooKeys, 150: 117-126. https://doi.org/10.3897//zookeys.150.2109

Baraloto C., 2003. Régénération forestière naturelle : de la graine à la jeune tige. Revue Forestière Française.LV, 179-187. https://doi.org/10.4267/2042/5770

Battandier J.A., 1914. Plantae paraguarienses novae. Bulletin de la Société Botanique de Genève, 2(6) : 1-252.

Benhouhou S., Yahi N., Véla E., 2018. Key Biodiversity Areas (KBAs) for plants in the Mediterranean region: Algeria. In: Valderrábano M., Gil T., Heywood V., Montmollin B. de (eds) Conserving wild plants in the south and east Mediterranean region: 53-60. Gland, Switzerland), Málaga, Spain, IUCN. https://doi.org/10.2305/IUCN.CH.2018.21.en

Bidou D., Guiraud P., 2012. Béton et biodiversité : une complicité à découvrir. Solutions béton, hors-série : $1-8$.

Bonanno G., 2013. Adaptive management as a tool to improve the conservation of endemic floras: the case of Sicily, Malta and their satellite islands. Biodiversity and conservation, 22(6-7): 1317-1354. http://dx.doi.org/10.1007/s10531-013-0473-9

Boulemtafes A., Hamel T., de Bélair G., Véla E., 2018. Nouvelles données sur la distribution et l'écologie de seize taxa végétaux du littoral de la péninsule de l'Edough (Nord-Est algérien). Bulletin de la Société linnéenne de Provence, 69 : 1-18.

Chaïd-Saoudi Y., 1987. Les Mammifères holocènes des gisements préhistoriques de Gueldaman-Akbou (Bédjaia), Columnata (Tiaret) et Ti-n Hanakaten (Djanet) en Algérie. Université Claude Bernard - Lyon 1, 1987.

Chenot J., Jaunatre R., Buisson E., Bureau F., Dutoit T., 2018. Impact of quarry exploitation and disuse on pedogenesis. Catena, $160: 354-365$.

http://dx.doi.org/10.1016/j.catena.2017.09.012

Chenot-Lescure J., Lescure F., 2019. Faut-il restaurer les carrières après exploitation ? Potentialités écologiques des carrières abandonnées à moyen terme (30-40 ans). Revue internationale d'écologie méditerranéenne, 45 : 5-34.

http://dx.doi.org/10.3406/ecmed.2019.2086

Daumas P., Quézel P., Santa S., 1952. Contribution à l'étude des groupements végétaux rupicoles d'Oranie. Bulletin de la Société d'Histoire Naturelle de l'Afrique du Nord, 43 : 186-202.

Davis P.H., 1951. Cliff vegetation in the eastern Mediterranean. The Journal of Ecology, 39(1): 63-93. http://dx.doi.org/10.2307/2256628

Debeaux J.O., Dautez G., 1889. Synopsis de la flore de Gibraltar. Paris, F. Savy. P39-40 
Bulletin de la Société Royale des Sciences de Liège, Vol. 90, Articles, 2021, p.101 - 124

Dobignard A. \& Chatelain C. (2011). Index synonymique de la flore d'Afrique du Nord. Volume 3 : Dicotyledoneae: Balsaminaceae-Euphorbiaceae. C.J.B. Genève

Dobrowski S.Z., 2011. A climatic basis for microrefugia: the influence of terrain on climate. Global Change Biology, 17(2): 1022-1035. http://dx.doi.org/10.1111/j.1365-2486.2010.02263.x

Fréville H., Colas B., Ronfort J., Riba M., Olivieri I., 1998. Predicting endemism from population structure of a widespread species: case study in Centaurea maculosa Lam. (Asteraceae). Conservation Biology, 12: 1269-1278. http://dx.doi.org/10.1046/j.1523-1739.1998.96483.x

García M.B., Domingo D., Pizarro M., Font X., Gómez D., Ehrlén J., 2020. Rocky habitats as microclimatic refuges for biodiversity, A close-up thermal approach. Environmental and Experimental Botany, 170: 103886. https://doi.org/10.1016/j.envexpbot.2019.103886

García M.B., Guzmán D., Goñi D., 2002. An evaluation of the status of five threatened plant species in the Pyrenees. Biological Conservation, 103(2): 151-161.

https://doi.org/10.1016/S0006-3207(01)00113-6

Hall F.C., 2002. Photo point monitoring handbook: Part A-field procedures. US Department of Agriculture, Forest Service, Pacific Northwest Research Station.

Hallal N., Dubois L., Bougdal R., Djouder F., 2018. Instabilités gravitaires dans la région de Béjaïa (Algérie) : Inventaire et appréciation de l'importance relative des différents paramètres conduisant au déclenchement, au maintien ou à l'activation des instabilités. Bulletin of Engineering Geology and the Environment, 77(2): 631-645.

http://dx.doi.org/10.1007/s10064-017-1050-3

Imbert E., Youssef S., Carbonell D., Baumel A., 2012. Do endemic species always have a low competitive ability? A test for two Mediterranean plant species under controlled conditions. Journal of Plant Ecology, 5(3): 305-312. http://dx.doi.org/10.1093/jpe/rtr033

Jeanmonod D., 1982. Nouvelle station du Silene aristidis Pomel en Algérie. Candollea, 37 : $485-495$.

Khennouf H., Chefrour A., Corcket E., Alard D., Véla E., 2018. La végétation dunaire du littoral de Jijel (Algérie) : proposition d'une nouvelle Zone Importante pour les Plantes. Revue d'écologie (La Terre et La Vie), 73(3) : 345-362.

Kherbouche F., 2015. Le néolithique tellien de la grotte de Gueldaman GLD1 (Babors d'Akbou, Algérie, VIII-V millénaire BP). Thèse de Doctorat en Archéologie et Préhistoire, Université Toulouse-Jean Jaurès, France. http://www.theses.fr/2015TOU20116/document

Larson D.W., Matthes U., Kelly P.E., 2005. Cliff ecology: pattern and process in cliff ecosystems. Cambridge, New York, Cambridge University Press.

http://dx.doi.org/10.1017/CBO9780511525582 
Bulletin de la Société Royale des Sciences de Liège, Vol. 90, Articles, 2021, p.101 - 124

Lavergne S., Thompson J. D., Garnier E., Debussche M., 2004. The biology and ecology of narrow endemic and widespread plants: a comparative study of trait variation in 20 congeneric pairs. Oikos, 107(3): 505-518.

http://dx.doi.org/10.1111/j.0030-1299.2004.13423.x

Lorite J., Serrano F., Lorenzo A., Cañadas E.M., Ballesteros M., Peñas J., 2017. Rock climbing alters plant species composition, cover, and richness in Mediterranean limestone cliffs. PloS one, 12(8), e0182414. https://doi.org/10.1371/journal.pone.0182414

Maire R., 1957-1987. Flore de L'Afrique du Nord, Vol. I -XVI. Paris, Lechevalier.

Maire R., 1963. Flore de L'Afrique du Nord, Vol. X. Paris, Lechevalier.

Mansouri S., Miara M.D., Hadjadj-Aoul S., 2018. État des connaissances et conservation de flore endémique dans la région d'Oran (Algérie occidentale). Acta Botanica Malacitana, 43: 23-30. http://dx.doi.org/10.24310/abm.v43i0.4361

Médail F. Diadema K., 2006. Biodiversité végétale méditerranéenne et anthropisation : approches macro et micro-régionales. Annales de géographie, 651(5): 618-640.

http://dx.doi.org/10.3917/ag.651.0618

Médail F., Diadema K., 2009. Glacial refugia influence plant diversity patterns in the Mediterranean Basin. Journal of biogeography, 36(7): 13331345. https://doi.org/10.1111/j.1365-2699.2008.02051.x

Médail F., Myers N., 2004. Mediterranean Basin. In: Mittermeier R.A., Robles Gil P., Hoffmann M., Pilgrim J., Brooks T., Mittermeier C.G., Lamoreux J., da Fonseca G.A.B. (eds) Hotspots revisited: Earth's biologically richest and most endangered terrestrial ecoregions: 144-147. CEMEX (Monterrey), Conservation International (Washington), Agrupacio'n Sierra Madre (Mexico).

Mesbah M., Bekdouche F., Véla E., Sahnoune M., 2019. Silene sessionis. The IUCN Red List of Threatened Species, 2019 (3): e.T72458759A72458763.

http://dx.doi.org/10.2305/IUCN.UK.2019-3.RLTS.T72458759A72458763.en

Mesbah M., Bekdouche F., Laidi K., Sahnoune M., Véla E., 2020a. Silene aristidis. The IUCN Red List of Threatened Species 2020: e.T135259613A135259617. https://dx.doi.org/10.2305/IUCN.UK.2020-2.RLTS.T135259613A135259617.en

Mesbah M., Bekdouche F., Laidi K., Sahnoune M., Véla E., 2020b. Silene auriculifolia. The IUCN Red List of Threatened Species 2020: e.T156127607A156127637. https://dx.doi.org/10.2305/IUCN.UK.2020-2.RLTS.T156127607A156127637.en

Montmollin De B., Strahm W. (eds), 2005. Le "Top 50" des plantes menacées des îles méditerranéennes : comment les sauver de l'extinction. Gland \& Cambridge, UICN. https://portals.iucn.org/library/sites/library/files/documents/2005-025-Fr.pdf 
Neves S.S., Watson M.F., 2004. Phylogenetic relationships in Bupleurum (Apiaceae) based on nuclear ribosomal DNA ITS sequence data. Annals of Botany, 93(4): 379-398. http://dx.doi.org/10.1093/aob/mch052

Nuzzo V.A., 1995. Effects of rock climbing on cliff goldenrod (Solidago sciaphila Steele) in northwest Illinois. American Midland Naturalist, 133(2): 229-241.

Panitsa M., Kontopanou A., 2017. Diversity of chasmophytes in the vascular flora of Greece: floristic analysis and phytogeographical patterns. Botanica Serbica, 41(2): 199-211. $\underline{10.5281 / \text { zenodo. } 1026483}$

Pomel, A. 1875. Nouveaux matériaux pour la flore atlantique. Fascicule 2. Bulletin de la Société des Sciences Physiques, Naturelles et Climatologiques de l'Algérie, 286.

Pomel M.A., 1888. Études sur des espèces barbaresques des types des Evax et des Filago. Bulletin de la Société Botanique de France, 35(6) : 333-337.

Purdy K., Wright I.A., 2019. Impact of concrete on riparian ecosystems. In IOP Conference Series: Earth and Environmental Science, 344: 012033.

http://dx.doi.org/10.1088/1755-1315/344/1/012033

Quézel P., 1957. Peuplement végétal des hautes montagnes de l'Afrique du Nord : essai de synthèse biogéographique et phytosociologique. In : Lechevalier P. (Ed.) Encyclopédie biogéographique et écologique, 10 : 1-445. Paris, P. Lechevalier.

Quézel P., 1985. Definition of the Mediterranean region and the origin of its flora. In: Gomez-Campo C. (éd.) Plant conservation in the Mediterranean area: 9-24. Heidelberg, Springer-Verlag.

Quézel P., Santa S., 1962-1963. Nouvelle flore de l'Algérie et des régions désertiques méridionales. Paris, CNRS.

Radford E.A., Catullo G., Montmollin B.de (eds.), 2011. Important Plant Areas of the south and east Mediterranean region: priority sites for conservation. Gland, Switzerland and Malaga, Spain, IUCN.

Saadi N., Benali L., 2015. Cartographie de la répartition et estimation de la taille de la population de Buplèvre à feuille de plantain (Bupleurum plantagineum Desf.) du littoral oriental de Djebel Gouraya - Petite Kabylie, Algérie. Mémoire de Master en Biologie de la conservation. Université de Bejaia, Algérie.

http://www.univ-bejaia.dz/jspui/handle/123456789/6909

Schmera D., Rusterholz H.-P., Baur A., Baur, B., 2018. Intensity-dependent impact of sport climbing on vascular plants and land snails on limestone cliffs. Biological Conservation, 224: 63-70. http://dx.doi.org/10.1016/j.biocon.2018.05.012 
Seltzer, P., Auberty E., 1946. Le climat de l'Algérie. Travaux de l'Institut de météorologie et de physique du globe de l'Algérie, hors sér., Université d'Alger. Alger, Imprimerie La TypoLitho et de J. Carbonel.

Silva J.L., Mejías J.A., García M.B., 2015. Demographic vulnerability in cliff-dwelling Sonchus species endemic to the western Mediterranean. Basic and Applied Ecology, 16(4): 316-324. https://doi.org/10.1016/j.baae.2015.02.009

Slimi A., Larue J.P., 2015. Réseau routier et risque d'éboulements rocheux et de chutes de blocs - cas du tronçon de la route nationale $\mathrm{n}^{\circ} 5$ dans les gorges de Lakhdaria (basse vallée de 1’Isser, Bouira, Algérie). Geomaghreb, 11:39-59.

Soubeyroux J.M., Kitova N., Blanchard M., Vidal J.P., Martin E., Dandin P., 2012. Caractérisation des sècheresses des sols en France et changement climatique : Résultats et applications du projet ClimSec. La Météorologie, 78: 21-30.

https://doi.org/10.4267/2042/47512

Strumia S., Buonanno M., Aronne G., Santo A., Santangelo A., 2020. Monitoring of plant species and communities on coastal cliffs: Is the use of unmanned aerial vehicles suitable? Diversity, 12(4): 149. http://dx.doi.org/10.3390/d12040149

Tassin C., 2012. Paysages végétaux du domaine méditerranéen : bassin méditerranéen, Californie, Chili central, Afrique du Sud, Australie méridionale. Paris, IRD éditions. http://dx.doi.org/10.4000/books.irdeditions.9781

UICN, 2001. Catégories et critères de l'UICN pour la Liste rouge, Version 3.1. Commission de la sauvegarde des espèces de l'UICN. Gland, Cambridge, UICN.

Véla E., Rebbas K., Lansdown R.V., 2019. Allium trichocnemis. The IUCN Red List of Threatened Species, 2019 (3): e.T13145944A18610859.

Véla, E., Benhouhou S., 2007. Évaluation d'un nouveau point chaud de biodiversité végétale dans le Bassin méditerranéen (Afrique du Nord). Comptes rendus biologies, 330(8): 589-605. http://dx.doi.org/10.1016/j.crvi.2007.04.006

Véla E., Magnin F., Pavon D., Pfenninger M., 2008. Phylogénie moléculaire et données paléobiogéographiques sur le gastéropode terrestre Tudorella sulcata (Draparnaud, 1805) en France et en Algérie orientale. Geodiversitas, 30(1) : 233-246.

Verlaque R., Médail F., Quézel P., Babinot J.F., 1997. Endémisme végétal et paléogéographie dans le bassin méditerranéen. Geobios, 30: 159-166.

http://dx.doi.org/10.1016/S0016-6995(97)80083-6

Walter K.S., Gillet H.J., 1998. 1997 IUCN red list of threatened plants. Gland, Switzerland, IUCN. 
Bulletin de la Société Royale des Sciences de Liège, Vol. 90, Articles, 2021, p.101 - 124

Wilhite D.A., Glantz M.H., 1985. Understanding: the drought phenomenon: the role of definitions. Water international, 10(3): 111-120.

https://doi.org/10.1080/02508068508686328

Yahi N., Véla E., Benhouhou S., De Belair G., Gharzouli, R., 2012. Identifying important plants areas (key biodiversity areas for plants) in Northern Algeria. Journal of threatened taxa, 4: 2753-2765. http://dx.doi.org/10.11609/JoTT.o2998.2753-65

Youssef S., Baumel A., Véla E., Juin M., Dumas E., Affre L., Tatoni T., 2011. Factors underlying the narrow distribution of the Mediterranean annual plant Arenaria provincialis (Caryophyllaceae). Folia Geobotanica, 46(4): 327-350.

http://dx.doi.org/10.1007/s12224-011-9101-1 